\title{
MKP-1 overexpression is associated with chemoresistance in bladder cancer via the MAPK pathway
}

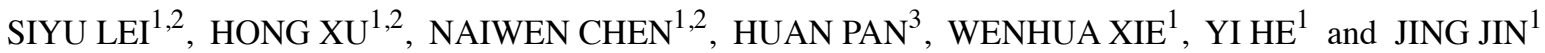 \\ ${ }^{1}$ Department of Urology, The First Affiliated Hospital of Jiaxing University, Jiaxing, Zhejiang 314000; \\ ${ }^{2}$ Department of Surgery, The 2nd Clinical Medical College of Zhejiang Chinese Medical University, Hangzhou, \\ Zhejiang 310000; ${ }^{3}$ Department of Central Laboratory, The First Affiliated Hospital of Jiaxing University, \\ Jiaxing, Zhejiang 314000, P.R. China
}

Received July 26, 2019; Accepted January 14, 2020

DOI: $10.3892 / 01.2020 .11741$

\begin{abstract}
Mitogen activated protein kinase phosphatase-1 (MKP-1) has been revealed to be overexpressed in bladder cancer, particularly in non-muscle invasive bladder cancer. MKP-1 may also be associated with chemotherapy resistance. However, the underlying mechanism is yet to be elucidated. The current study investigated the expression of MKP-1 by performing immunohistochemistry in surgically resected specimens obtained from primary and recurrent patients with bladder cancer. The results revealed that MKP-1 expression increased in recurrent patients. Additionally, a 3D model of the human bladder cancer cell line, RT112, was established to determine the role of MKP-1 in drug resistance. The results demonstrated that MKP-1 overexpression protected bladder cancer cells against cell death. Contrarily, MKP-1 knockdown was revealed to sensitize cells to death. In addition, the application of MAPK inhibitors effectively increased RT112 cell sensitivity to pirarubicin. In conclusion, the results of the current study indicated that MKP-1 treatment resulted in bladder cancer cell chemoresistance via JNK, ERK and p38
\end{abstract}

Correspondence to: Professor Yi He or Ms. Jing Jin, Department of Urology, The First Affiliated Hospital of Jiaxing University, 1882 Zhonghuan South Road, Nanhu, Jiaxing, Zhejiang 314000, P.R. China

E-mail: 84748480@qq.com

E-mail: jxjjnicole@163.com

Abbreviations: MKP-1, mitogen activated protein kinase phosphatase-1; THP, Pirarubicin; FGFR3, fibroblast growth factor 3; NMIBC, non-muscle invasive bladder cancer; MIBC, muscle invasive bladder cancer; TURBT, transurethral resection of the bladder tumor; ERK, extracellular signal-regulated kinase; JNK, c-Jun NH2-terminal kinase; MAPK, mitogen activated protein kinases; MKPs, MAPK phosphatases

Key words: mitogen activated protein kinase phosphatase-1, fibroblast growth factor 3, resistance, bladder cancer, 3D model, RT112 cell pathways. MKP-1 may also serve as a potential therapeutic target for chemoresistance in patients with bladder cancer.

\section{Introduction}

Globally, bladder cancer has the 9th highest and 14th highest rates of incidence and mortality of all types of cancer (1). Approximately 900,000 patients are newly diagnosed each year, with 250,000 deaths occurring in the same timeframe (2). Of all newly diagnosed cases of bladder cancer, $\sim 75 \%$ are non-muscle invasive (NMIBC) and $\sim 25 \%$ are muscle invasive (MIBC). The transurethral resection of bladder tumors combined with intravesical instillation has become the first-line treatment for high-risk patients with NMIBC. Instillation therapy is usually applied with Pirarubicin (THP), Epirubicin and Mitomycin (3), with THP being the most commonly applied, clinically. It is generally accepted that THP kills tumor cells by inducing cell apoptosis. However, $>30 \%$ of patients with NMIBC still exhibit recurrence or the development of MIBC within 5 years (4). Chemoresistance is a serious challenge to bladder tumor therapy. Therefore, it is necessary to identify more effective targets to better cope with chemotherapeutic resistance.

Fibroblast growth factor receptor 3 (FGFR3) is a member of the FGFR family and is implicated in various cellular activities, including proliferation, migration, survival and death (5). FGFR3 mutations are common in bladder cancer, occurring in $\sim 75 \%$ of patients with NMIBC. FGFR3 is therefore an attractive target for the treatment of bladder cancer $(6,7)$. It has been determined that FGFR3 mutations are correlated with FGFR3 overexpression (8). Further study has revealed that $74 \%$ of patients with bladder cancer exhibiting a high expression of FGFR3 also possessed an FGFR3 mutation, indicating that FGFR3 expression is strongly associated with FGFR3 mutation (9). Clinically, chemoresistance occurs frequently in patients with bladder cancer that exhibit an aberrant activation of FGFR3. It has been found that chemoresistance may be associated with FGFR3 mutations (10). It has also been demonstrated that FGFR3 expression is increased in metastatic tumors compared with primary bladder cancer (11). It can therefore be hypothesized that FGFR3 overexpression may be involved in chemoresistance. Furthermore, resistance 
commonly occurs as a result of extensive phosphorylation, which is activated by FGFR3 downstream signaling. FGFR3 activates multiple downstream signaling pathways, including MAPK, PI3K/AKT and STAT pathways $(7,12,13)$.

MKP-1 is a member of the MKP family that negatively regulates the MAPK pathway (14). It also serves a significant role in proliferation, inflammation and apoptosis $(15,16)$, and is involved in the MAPK pathway. Its dephosphorylation inactivates JNK, ERK and p38 (17,18). MKP-1 is overexpressesed in various types of epithelial cancer at early stages, including gastric, bladder, prostate and colon carcinomas $(19,20)$. However, MKP-1 expression also decreases as tumor grades and stages advance $(21,22)$, indicating that MKP-1 is an essential factor for the determination of benign and malignant tumors. An increasing number of studies have revealed that MKP-1 correlates with drug resistance in lung cancer, ovarian cancer, breast cancer, osteosarcoma and lymphoma (23-26). MKP-1 has also been revealed to be overexpressed in NMIBC. However, whether MKP-1 is associated with chemoresistance in bladder cancer is yet to be fully elucidated. Furthermore, whether an association exists between FGFR3 and MKP-1 in the regulation of chemoresistance in bladder cancer is yet to be determined. The present study assessed FGFR3 and MKP-1 in patients with primary and recurrent bladder cancer, and established a 3D model to determine the role of MKP-1 in chemoresistance. The results indicated that MKP-1 inhibited RT112 cell apoptosis, which served an important role in the chemotherapeutic resistance of bladder cancer.

\section{Materials and methods}

Tissue specimens. Bladder cancer tissues, which were resected by urologists, were obtained from patients (age range, 47-83 years) between March 2018 and February 2019. A total of 10 specimens (5 primary and 5 recurrent urothelial carcinoma specimens) were diagnosed as urothelial carcinoma in accordance with the histological criteria of the World Health Organization, and 5 recurrent patients all had a history of vesical chemotherapy instillation of pirarubicin. Following resection, samples were immediately and stored at $-80^{\circ} \mathrm{C}$ until further analysis. The clinical characteristics of the patients included in the current study are presented in Table I. The present study was approved by the Ethics Committee of The First Affiliated Hospital of Jiaxing University, and informed consent was obtained from each patient.

Cells, culture and reagents. The human bladder cancer cell line RT112 was purchased from the Leibniz Institute DSMZ and maintained in RPMI 1640 medium supplemented with $10 \%$ fetal bovine serum at $37^{\circ} \mathrm{C}$ in a humidified atmosphere containing of $5 \% \mathrm{CO}_{2}$. Tissue culture media and serum were purchased from Hyclone (GE Healthcare Life Sciences). 3D Cell Culture Gel (cat. no. P720M-10) was purchased from Col-Tgel Med (http://www.101bio.com/P720_3D_cell_ culture_gel.php). Rabbit polyclonal antibodies against total and phosphorylated JNK, ERK1/2 and p38 were purchased from Cell Signaling Technology, Inc. The JNK inhibitor SP600125 (cat. no. T3109), the ERK inhibitor PD98059 (cat. no. T2623), the p38 inhibitor SB202190 (cat. no. T2301) and THP were purchased from Target Molecule Corp.
Small interfering RNA (siRNA) transfection. MKP-1 siRNA and scrambled siRNA were purchased from GE Healthcare Dharmacon, Inc. The sequences were as follows: MKP-1 siRNA forward, 5'-CACAAGGCAGACATCAGCTC-3' and reverse, 5'-AGGTAAGCAAGGCAGATGGT-3'; scrambled siRNA forward, 5'-GGGTGTGAACCATGAGAAGT-3' and reverse, 5'-GACTGTGGTCATGAGTCCT-3'. RT112 cells were transiently transfected with $100 \mathrm{nM}$ MKP-1 siRNA and scrambled siRNA in six-well plates treated with TurboFect reagent according to manufacturer's protocol. After $48 \mathrm{~h}$, cells were harvested for subsequent experimentation.

Immunohistochemistry. Fixed tissues were dehydrated, embedded, sliced and dyed, and washed in triplicate. Tissue sections were subsequently autoclaved in sodium citrate buffer ( $\mathrm{pH}$ 6.0) for $30 \mathrm{~min}$, after which goat serum blocking solution was added and samples were incubated. Primary rabbit polyclonal anti-MKP-1 antibodies (dilution: 1:500, catlog: NBP2-67909, Novus Biologicals, LLC) were then added and incubated overnight at $4^{\circ} \mathrm{C}$. After being washed three times, samples were further incubated with biotinylated secondary antibodies (the Jackson laboratory, Bar Harbor, ME, USA) for $30 \mathrm{~min}$ at room temperature. Sections were then developed using Dimethyl benzidine for $2 \mathrm{~min}$. Slides were counterstained with hematoxylin and examined using light microscopy. The labeled substance appeared yellow-brown under the microscope. Negative control sections were not incubated with primary antibodies. However, the remaining steps of the protocol were the same.

Reverse transcription-quantitative PCR (RT-qPCR). Cells were lysed and total RNA was isolated using TRIzol ${ }^{\circledR}$ reagent (Invitrogen; Thermo Fisher Scientific, Inc.) according to manufacturer's protocol. RNA was reverse transcribed using the M-MLV reverse transcription kit (Takara, Tokyo, Japan). Quantitative PCR was performed using a SYBR Premix Ex Taq kit (Roche, Basel, Switzerland). The sequences of the primers obtained from Sangon Biotech Co., Ltd. were as follows: MKP-1 forward, 5-CCTTTCTGTACCTGGGCA GT-3 and reverse, 5-GGTTGGGACAATTGGCTGAG-3; GAPDH forward, 5-GGGTGTGAACCATGAGAAGT-3 and reverse, 5-GACTGTGGTCATGAGTCCT-3. GAPDH was used as an internal control. The thermocycling conditions for qPCR were as follows: $94^{\circ} \mathrm{C}$ for $2 \mathrm{~min}$, followed by 40 cycles at $94^{\circ} \mathrm{C}$ for $15 \mathrm{sec}, 60^{\circ} \mathrm{C}$ for $1 \mathrm{~min}$ and $72^{\circ} \mathrm{C}$ for $10 \mathrm{~min}$. The $2^{-\Delta \Delta \mathrm{Cq}}$ analysis method was used to calculate relative expression.

Flow cytometry (FACS). Apoptosis was assessed using an Annexin V-APC kit (BD Biosciences) in accordance with the manufacturer's protocol. Treated cells were washed and centrifuged $\left(1,000 \mathrm{rpm}, 10 \mathrm{~min}, 4^{\circ} \mathrm{C}\right)$. The supernatant was resuspended and $500 \mu \mathrm{l}$ binding buffer, $2 \mu \mathrm{l}$ Annexin V-APC and $5 \mu \mathrm{l}$ propidium iodide was added. Cells were analyzed using a FACS Canto plus flow cytometer (BD Biosciences) after 15 min of incubation.

Western blot analysis. Western blot analysis was performed as previously described (27). Cells were harvested, washed and centrifuged $\left(1,500 \mathrm{rpm}, 5 \mathrm{~min}, 4^{\circ} \mathrm{C}\right)$. An equal quantity of protein was loaded on 10 or $12 \%$ SDS-PAGE gels and 
Table I. Characteristics of bladder cancer tissue samples from 10 Chinese patients.

\begin{tabular}{clccccc}
\hline Patient no. & Sex & Age, years & $\begin{array}{c}\text { Histological } \\
\text { typing WHO }\end{array}$ & Grade & $\begin{array}{c}\text { Primary or } \\
\text { recurrent }\end{array}$ & $\begin{array}{c}\text { History of } \\
\text { chemotherapy }\end{array}$ \\
\hline 1 & Female & 82 & Urothelial carcinoma & Low grade & Primary & No \\
2 & Male & 68 & Urothelial carcinoma & Low grade & Primary & No \\
3 & Female & 68 & Urothelial carcinoma & Low grade & Primary & No \\
4 & Male & 77 & Urothelial carcinoma & Low grade & Primary & No \\
5 & Female & 47 & Urothelial carcinoma & Low grade & Primary & No \\
6 & Female & 69 & Urothelial carcinoma & Low grade & Recurrent & Pirarubicin \\
7 & Male & 83 & Urothelial carcinoma & Low grade & Recurrent & Pirarubicin \\
8 & Female & 81 & Urothelial carcinoma & Low grade & Recurrent & Pirarubicin \\
9 & Male & 64 & Urothelial carcinoma & Low grade & Recurrent & Pirarubicin \\
10 & Male & 59 & Urothelial carcinoma & Low grade & Recurrent & Pirarubicin \\
\hline
\end{tabular}

WHO, World Health Organization.

transferred onto nitrocellulose membranes. Membranes were subsequently blocked with $5 \%$ skimmed milk and then hybridized to primary antibodies overnight at $4^{\circ} \mathrm{C}$. Subsequently, samples were incubated with secondary antibodies for $1 \mathrm{~h}$ at room temperature. Bound antibodies was detected using an enhanced chemiluminescence reagent in accordance with the to manufacturer's protocol. GAPDH and actin were used as a loading control.

Cell viability assay. Cell viability was determined using a cell counting kit 8 (CCK8) assay. Freshly isolated cells (5x10 $3 /$ well) were seeded into 96-well plates. Cells were pretreated with SP600125 $(20 \mu \mathrm{mol} / \mathrm{l})$, PD98059 $(20 \mu \mathrm{mol} / \mathrm{l})$ and SB202190 ( $25 \mu \mathrm{mol} / \mathrm{l})$ for $1 \mathrm{~h}$, after which THP $(80 \mathrm{nmol} / \mathrm{l})$ was added to each group. After $48 \mathrm{~h}, 10 \mu \mathrm{l} \mathrm{CCK} 8$ (Beyotime Institute of Biotechnology) was added to cells and the optical density of each well was measured at $450 \mathrm{~nm}$ using an ELISA reader (BioTek, Winooski, Vermont, USA) according to the manufacturer's protocol.

Statistical analysis. Statistical analysis was performed using the Statistical Package of Social Sciences (IBM Corp.; version 20.0.). Statistical significance was determined using One-way ANOVA and followed by Tukey post-hoc tests across the groups. Differences between the two groups were analyzed by Unpaired t-test. Data were expressed as the mean \pm standard deviation and $\mathrm{P}<0.05$ was considered to indicate a statistically significant difference.

\section{Results}

FGFR3 and MKP-1 expression is increased in recurrent bladder cancer cells. FGFR3 mutations or its expression commonly occurs in low grade, superficial bladder tumors $(8,28)$. FGFR3 also regulates cellular processes primarily via the MAPK pathway (13). MKP-1 has been proven to be associated with chemoresistance in many different types of tumor, and negatively regulates MAPKs. To determine if FGFR3 and MKP-1 are associated with chemoresistance in bladder cancer, the current study collected multiple specimens of primary and recurrent bladder cancer tissue. FGFR3 and MKP-1 expression were subsequently detected using immunohistochemistry (Fig. 1A). the results revealed that FGFR3 and MKP-1 expressions were increased in patients with recurrent cancer (Fig. 1B and C). Furthermore, MKP-1 mRNA levels (Fig. 1D) were increased in patients with recurrent bladder cancer. The results indicated that MKP-1 and FGFR3 expressions were upregulated in recurrent tissue, leading to the hypothesis that FGFR3 and MKP-1 may be closely associated with chemoresistance in human bladder cancer. FGFR3 and MKP-1 may therefore serve as potential targets for resistance therapy in patients with bladder cancer.

Establishment of human 3D bladder cancer model and role of MKP-1 in MAPK pathway. To better mimic the development of human bladder cancer, a novel 3D cellular model was established in the current study using RT112 cells in accordance with a previously published method (29). To explore the effect of MKP-1 in bladder cancer, RT112 cells were transiently transfected with scrambled siRNA (siNC) and MKP-1 siRNA (siMKP-1). The results revealed that MKP-1 mRNA levels (Fig. 2A) were significantly decreased in the siMKP-1 group, demonstrating that MKP-1 was effectively inhibited by MKP-1 siRNA. RT112 cells of the 2D and 3D model appeared to have different morphological characteristics (Fig. 2B). Specifically, $3 \mathrm{D}$ cells generated spheroids with a smooth surface and rounded shape. However, 2D cells appeared irregularly shaped with an obscure margin. The successful establishment of the $3 \mathrm{D}$ model was used for the subsequent 3D culture of remaining experiments.

MKP-1 serves an important role in the regulation of MAPK signaling by inactivating JNK, ERK and p38 (30). Thus, the current study hypothesized that MKP-1 knockdown may activate MAPK. As presented in Fig. 2C, no significant differences were identified between total levels of JNK, ERK1/2 and p38. Furthermore, levels of JNK, ERK1/2 and p38 phosphorylation were markedly increased in the siMKP-1 group, indicating that MKP-1 inhibited the phosphorylation of JNK, ERK1/2 and p38. The results indicated that MKP-1 serves an essential role in the negative regulation of JNK, ERK1/2 and p38 in RT112 cells. 
A

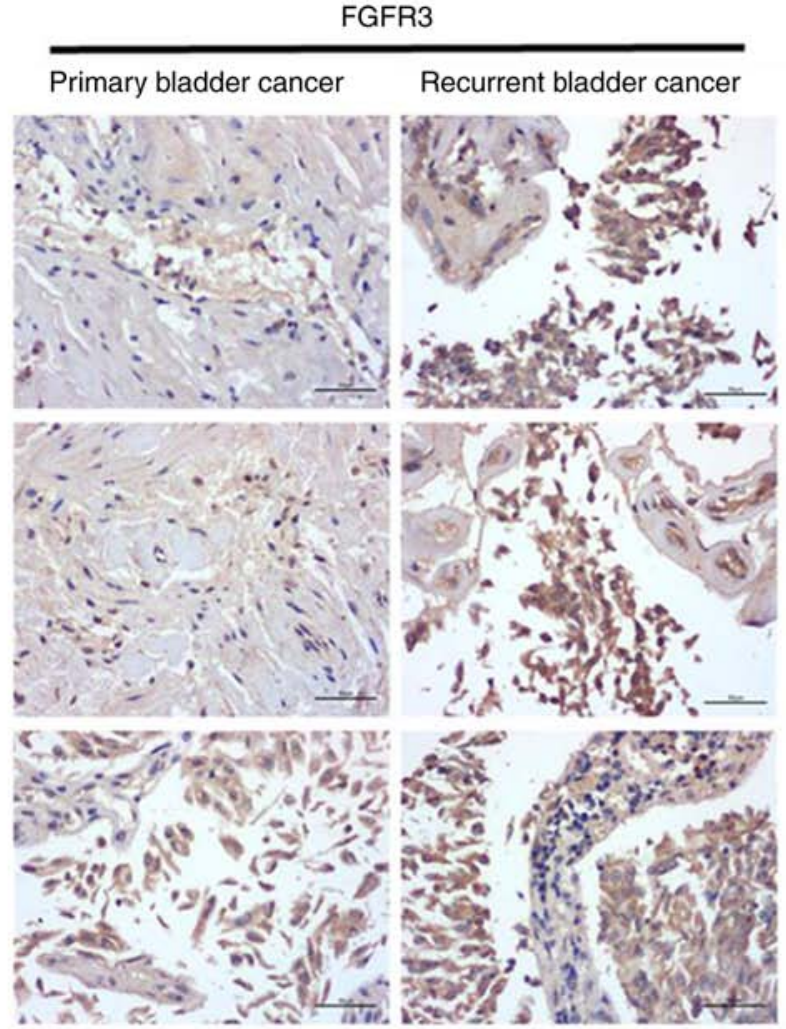

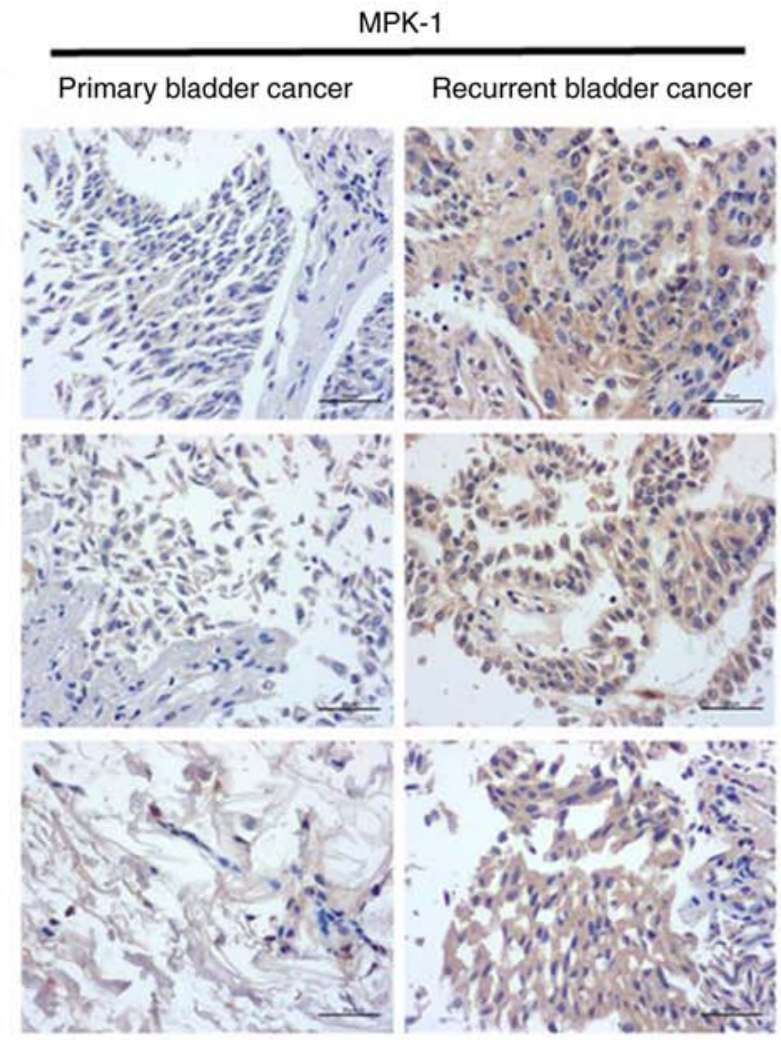
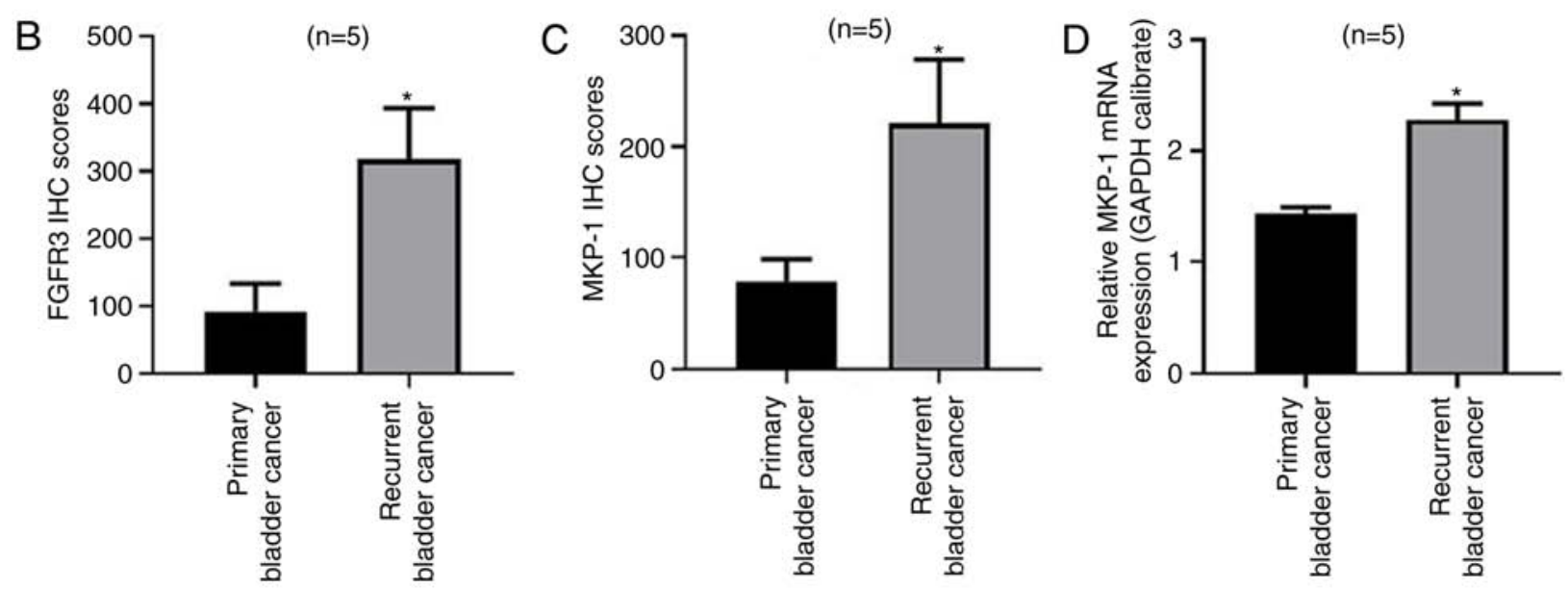

Figure 1. FGFR3 and MKP-1 expression levels are increased in recurrent bladder cancer tissue. (A) Immunohistochemical staining of primary and recurrent bladder cancer tissues was performed to detect FGFR3 and MKP-1 expression in representative patients. Scale bar, $100 \mu \mathrm{m}$. (B) Quantitative FGFR3 expression was analyzed using the Image-pro Plus 6.0 system. Histograms represent the mean density of FGFR3. (C) Quantitative MKP-1 expression was analyzed using the Image-pro Plus 6.0 system. Histograms represent the mean density of MKP-1. (D) Relative MKP-1 expression was determined by reverse transcription-quantitative PCR. " $\mathrm{P}<0.05$. FGFR3, fibroblast growth factor receptor 3; MKP-1, mitogen activated protein kinase phosphatase-1; IHC, immunohistochemistry.

MKP-1 affects drug susceptibility and protects RT112 cells from cell apoptosis. MKP-1 has been implicated in proliferation, survival, apoptosis and other cellular processes, and has also been correlated with chemoresistance (23). The current study aimed to establish the effects of MKP-1 knockdown on chemotherapeutics and to ascertain whether MKP-1 serves a role in bladder cancer drug resistance. The current study therefore treated siNC and siMKP-1 cells with gradually increasing doses of THP for $24 \mathrm{~h}$ (Fig. 3A). The results revealed that the viability of siMKP-1 cells was less than siNC cells, indicating that siMKP-1 cells were more sensitive to THP. As presented in Fig. 3A, the viability of siNC cells following THP ( $80 \mathrm{nmol} / \mathrm{l})$ treatment was significantly higher when compared with siMKP-1 cells. Extensive exposure of THP for 48 and $72 \mathrm{~h}$ (Fig. 3B) demonstrated that viability was visibly decreased in siMKP-1 cells. Two groups of cells were then selected and treated with $80 \mathrm{nmol} / \mathrm{l} \mathrm{THP}$ for $48 \mathrm{~h}$ to detect cell apoptosis via FCAS. The results revealed that cell apoptosis sharply increased in siMKP-1 cells (Fig. 3C and D), indicating that MKP-1 effectively inhibited RT112 cell apoptosis. Taken together, the results suggested that MKP-1 protected RT112 cells from cell apoptosis and served an important role in chemoresistance. 
A

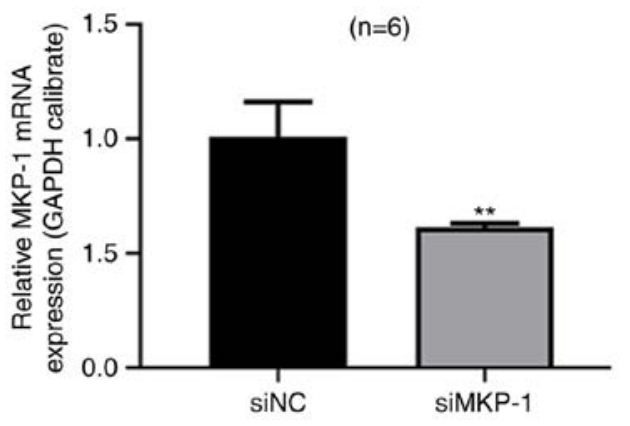

C

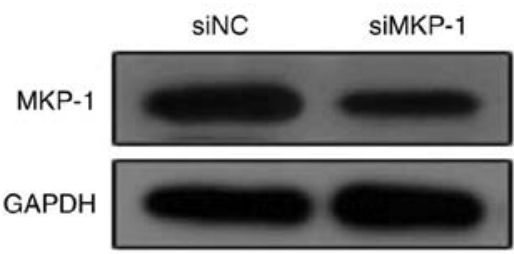

E

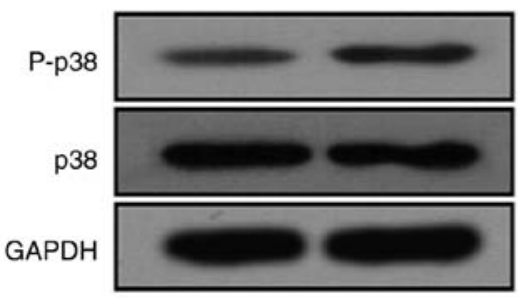

B
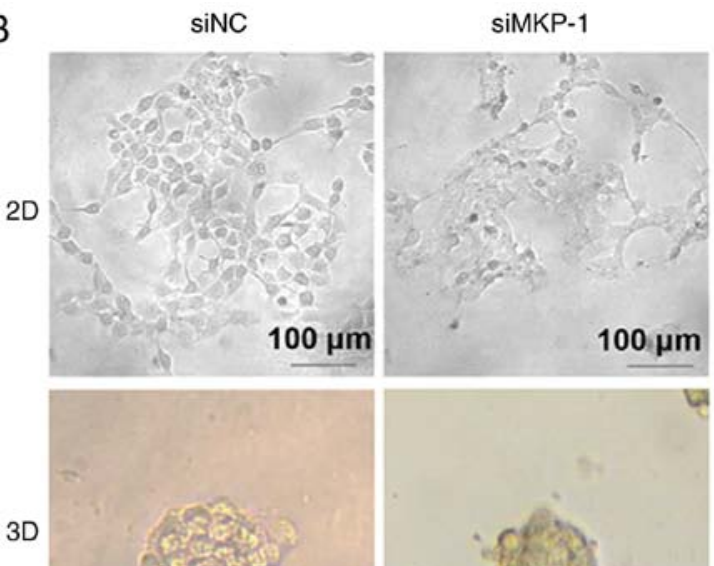

$3 D$
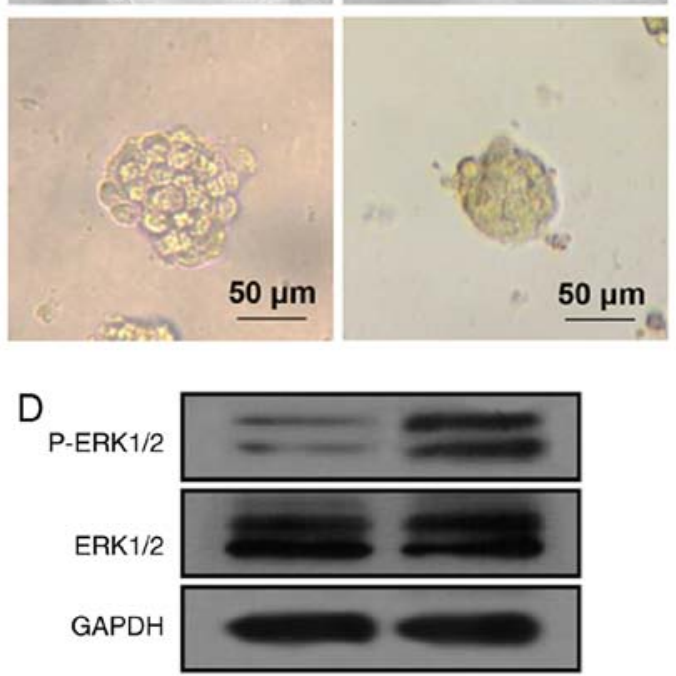

F

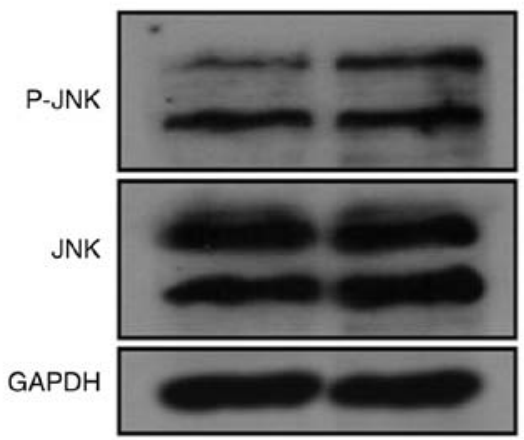

Figure 2. MKP-1 and MAPK expression in RT112 cells transfected with NC and MKP-1 siRNA. (A) Relative MKP-1 expression in the siNC and siMKP-1 groups was examined using reverse transcription-quantitative PCR. ${ }^{* *} \mathrm{P}<0.01$. (B) Representative microscopic images of siNC and siMKP-1 treated cells captured in both 2D and 3D environments under a phase contrast microscope. (C) MKP-1 expression of the siNC and siMKP-1 groups, as determined via western blotting. GAPDH was used as the internal control. (D) Phosphorylated and total ERK1/2 protein expressions of the siNC and siMKP-1 group, as determined via western blotting. GAPDH was used as the internal control. (E) Phosphorylated and total p38 protein expressions of the siNC and siMKP-1 group, as determined via western blotting. GAPDH was used as the internal control. (F) Phosphorylated and total JNK protein expression levels of the siNC and siMKP-1 group, as determined via western blotting. GAPDH was used as the internal control. MKP-1, mitogen activated protein kinase phosphatase-1; NC, negative control; siMKP-1, MKP-1 small interfering RNA; siNC, small interfering negative control.

$M K P-1$ is involved in chemoresistance via JNK, ERK and p38 pathways. MKP-1 knockdown induced cell apoptosis (Fig. 3C and D). However, the underlying mechanism of this process remains unclear. As aforementioned, the current study determined that JNK, ERK and p38 were upregulated in siMKP-1 cells (Fig. 2C), leading to the further hypothesis that JNK, ERK and p38 were responsible for cell apoptosis. To test this hypothesis, siNC and siMKP-1 groups were individually treated with JNK, ERK and p38 inhibitors (SP600125, PD98059 and SB202190, respectively). As presented in Fig. 4A, cell viability markedly increased in siMKP-1 cells treated with SP600125 and THP when compared with THP treatment alone. This indicated that SP600125 served a role in protecting siMKP-1 cells from cell death. PD98059 and
SB202190 exerted similar effects following treatment with SP600125. The results also revealed that SP600125 treatment inhibited JNK phosphorylation in siNC and siMKP-1 cells (Fig. 4B). Treatment with ERK or p38 inhibitors exerted similar effects. The results of Fig. 4 indicated that JNK, ERK and p38 serve significant roles in RT112 cell death. In conclusion, the results suggested that MKP-1 may serve a role in chemoresistance through JNK, ERK and p38 pathways in bladder cancer.

\section{Discussion}

Chemotherapy is a primary treatment of bladder cancer, and patients exhibiting chemoresistance have high recurrence 

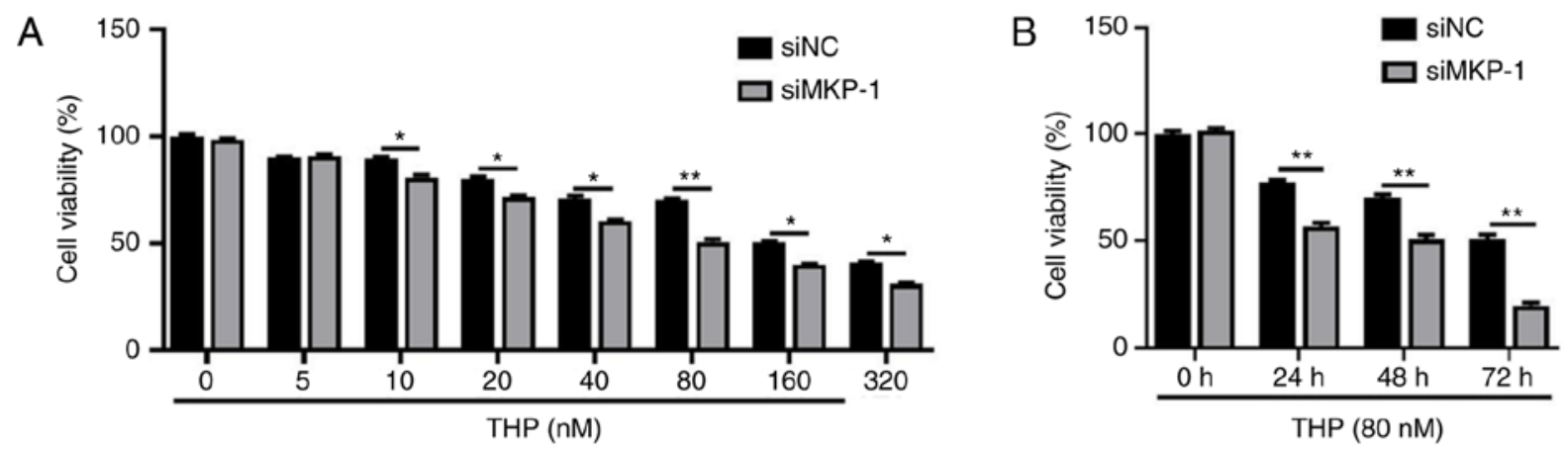

C
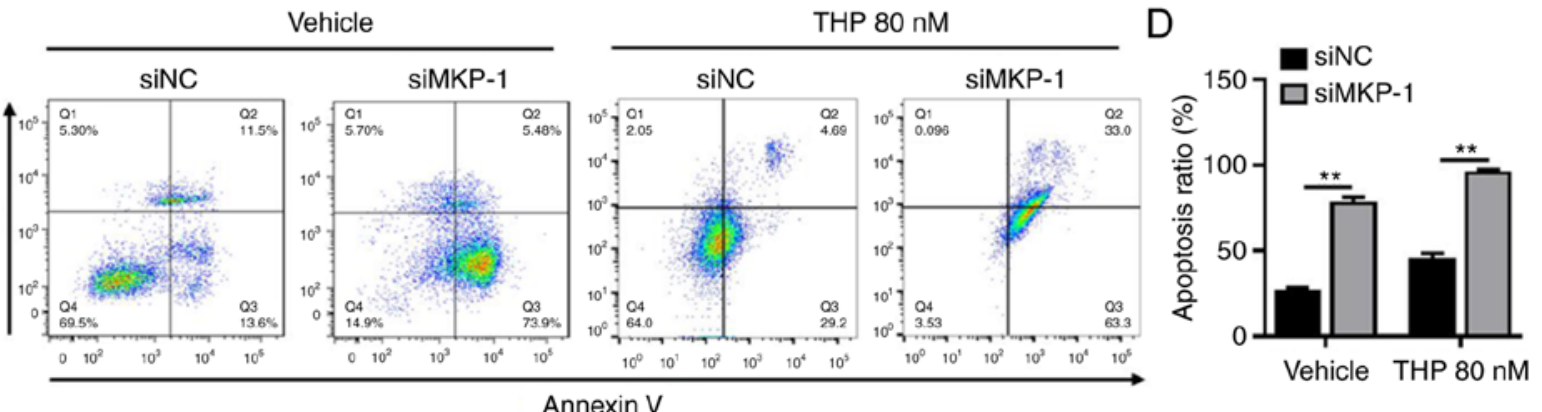

Figure 3. MKP-1 knockdown increases drug-susceptibility and induces cell apoptosis. Dose responses and the timed course of cell death in siNC and siMKP-1 treated cells is presented. (A) Two groups of cells were treated with increasing concentrations $(0,5,10,20,40,80,160$ and $320 \mathrm{nmol} / \mathrm{l})$ of THP for $24 \mathrm{~h}$. (B) Two groups of cells were treated with $80 \mathrm{nmol} / 1 \mathrm{THP}$ and incubated for different durations (24, 48 and $72 \mathrm{~h}$ ). Cell viability was determined via Cell Counting Kit-8 assays. ${ }^{*} \mathrm{P}<0.05$ and ${ }^{* *} \mathrm{P}<0.01$. (C) Cell apoptosis of the two groups was measured via flow cytometry with or without $80 \mathrm{nmol} / 1 \mathrm{THP}$ treatment. (D) A comparison of apoptosis rates between the two groups is presented. ${ }^{* *} \mathrm{P}<0.01$. MKP-1, mitogen activated protein kinase phosphatase-1; siNC, small interfering negative control; siMKP-1, MKP-1 small interfering RNA; THP, pirarubicin.
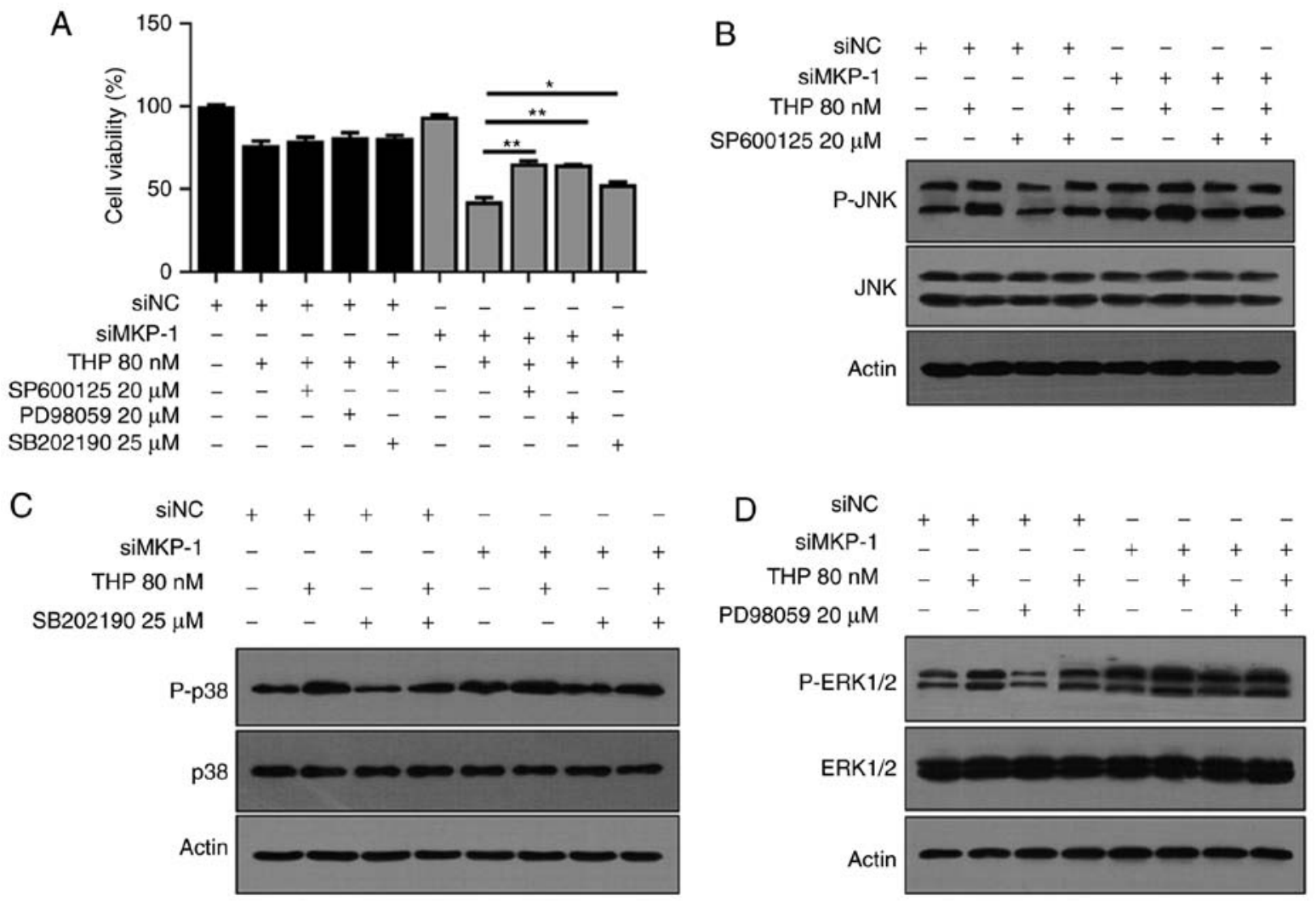

Figure 4. Effect of MAPK inhibitors on cell death. (A) siNC and siMKP-1 groups were left untreated or pretreated with SP600125, PD98059 and SB202190. Subsequently, samples were treated with $80 \mathrm{nmol} / 1$ THP. Cell viability was determined via Cell Counting Kit- 8 assays. "P<0.05 and ${ }^{* *} \mathrm{P}<0.01$. (B) Levels of phosphorylated and total JNK protein were determined via western blotting. Actin was used as the internal control. (C) Levels of phosphorylated and total p38 protein were determined via western blotting. Actin was used as the internal control. (D) Levels of phosphorylated and total ERK1/2 protein were determined via western blotting. Actin was used as the internal control. siNC, small interfering negative control; siMKP-1, MKP-1 small interfering RNA; THP, pirarubicin. 
rates, making it a major challenge to the treatment and prognosis of patients with bladder cancer (31). There are various mechanisms that have led to the emergence of chemoresistance, which involve diverse cell signaling pathways that are often interconnected, hindering bladder cancer therapy. At present, more studies aiming to identify various molecular targets have been intitated to create more effective methods of treating bladder cancer chemoresistance.

Many cell-based resistance assays are performed using conventional 2D models (32). However, in vivo cells exist in a 3D environment, which comprises the extracellular matrix and other neighboring cells. 3D models also mimic natural cellular responses in vivo, supporting angiogenesis, slowing proliferation, enhancing metastatic potential and increasing chemotherapeutic resistance $(33,34)$. Thus, the current study established a novel 3D bladder cancer cell model in vitro using gel-embedding methods.

FGFR3 is expressed on different cells and regulates biological processes (5) by triggering multiple signal transduction pathways, including MAPK, PI3K/AKT and JAK/STAT pathways (5). It has been demonstrated that FGFR3 mutations occur in myeloma, cervix and bladder cancer $(35,36)$, and is the most frequently mutated oncogene in NMIBC, primarily occurring in exons 7, 10 and 15 (37). Further studies have elucidate the FGFR3 overexpression and mutations are correlated $(8,9)$. It has been demonstrated that FGFR3 is highly expressed in patients with recurrent metastatic bladder cancer $(11,38)$. Similar results were obtained in the current study, indicating that results may be associated with chemoresistance (39). The dysregulated activity of FGFR3 may also mediate chemoresistance via gene mutations (40).

MKP-1, negatively regulates MAPKs through threonine or tyrosine residues. It has been demonstrated that MKP-1 expression is increased in lung, ovarian and breast cancer (23-25) after chemotherapy, which indicates that MKP-1 is closely associated with chemoresistance. MKP-1 has been demonstrated to exert sustaining tamoxifen resistance in breast cancer (41) and may induce resistance by inhibiting cell apoptosis $(26,42)$. FGFR3 is thought to regulate cell growth and survival by activating ERK (43), which in turn increases the expression of MKP-1 (44). MKP-1 is therefore regulated by FGFR3 to a certain extent. In the current study, it was determined that FGFR3 and MKP-1 expressions increased in patients with recurrent bladder cancer, and it was inferred that MKP-1 may serve as a novel target for the treatment of patients with resistant bladder cancer with FGFR3 overexpression. We must confess that such a small sample size may become a potential limitation of the study, but more samples will be collected for verification later, and future research will be carried out.

MKP-1 regulates the MAPK pathway by inactivating JNK, ERK and p38. However, previous studies have revealed that p38 and JNK are preferred substrates during cellular responses to stress $(45,46)$. A second study revealed that MKP-1 mediates cisplatin-induced apoptosis via the JNK pathway in lung cancer, but not via ERK or p38 pathways. Therefore, JNK, ERK and p38 may be regulated by MKP-1 depending on the type of cell and stimulus. The present study determined that JNK, ERK and p38 expression were increased in siMKP-1 cells, indicating that MKP-1 knockdown markedly activates JNK, ERK and p38 expression.
MKP-1 sensitizes RT112 cells to drugs and MKP-1 knockdown enhances THP-susceptibility. The results of the current study indicated that MKP-1 protected RT112 cells from apoptosis and that MKP-1 knockdown induced cell apoptosis, which indicated that MKP-1 was closely associated with chemoresistance. Corresponding JNK, ERK and p38 inhibitors were subsequently selected to block corresponding signal expression. The results revealed that JNK, ERK, p38 knockdown protects siMKP-1 cells from death, which strongly suggested that MKP-1 may inhibit RT112 cell death.

In conclusion, overexpression of MKP-1 protected cells from death and the knockdown of MKP-1 induced RT112 cell death. It was further revealed that the activation of JNK, ERK and p38 serve important roles in the regulation of cell apoptosis. MKP-1 may be involved in chemoresistance by inactivating JNK, ERK and p38, and may therefore lead to the inhibition of apoptosis in bladder cancer. Therefore, MKP-1 may serve as an effective therapeutic target for overcoming resistance in bladder cancer. However, further study is required to determine which upstream signaling pathways are involved and their specific roles in MAPK pathway, VEGFR and ROS (47) may become potential and great future research directions.

\section{Acknowledgements}

Not applicable.

\section{Funding}

The present study was supported by Jiaxing Science and Technology Project (grant. nos. 2017AY33004 and 2018AD32083) and Medical Scientific Research Foundation of Zhejiang Province, China (grant. no. 2019KY694).

\section{Availability of data and materials}

The datasets used and/or analyzed during the present study are available from the corresponding author on reasonable request.

\section{Authors' contributions}

SL, YH and JJ conceived and designed the study. HX, NC and WX collected tissue samples and patient data. SL, HX, NC, WX and HP performed the experiments and the statistical analyses. SL and XH analyzed the data and wrote the manuscript. YH and JJ reviewed and revised the manuscript. All authors read and approved the final manuscript.

\section{Ethics approval and consent to participate}

The present study was approved by the Ethics Committee of The First Hospital of The First Affiliated Hospital of Jiaxing University (approval no. 2016-056), and informed consent was obtained from all patients.

\section{Patient consent for publication}

Not applicable. 


\section{Competing interests}

The authors declare that they have no competing interests.

\section{References}

1. Mahdavifar N, Ghoncheh M, Pakzad R, Momenimovahed Z and Salehiniya H: Epidemiology, incidence and mortality of bladder cancer and their relationship with the development index in the world. Asian Pac J Cancer Prev 17: 381-386, 2016.

2. Klotz L and Brausi MA: World urologic oncology federation bladder cancer prevention program: A global initiative. Urol Oncol 33: 25-29,2015.

3. Liu S, Hou J, Zhang H, Wu Y, Hu M, Zhang L, Xu J, Na R, Jiang $\mathrm{H}$ and Ding Q: The evaluation of the risk factors for non-muscle invasive bladder cancer (NMIBC) recurrence after transurethral resection (TURBt) in Chinese population. PLoS One 10: e0123617, 2014

4. Miki T, Nonomura N, Kojima Y, Okuyama A, Nakano E, Kiyohara H, Fujioka H, Koide T, Wakatsuki A, Kuroda H, et al: A randomized study on intravesical pirarubicin (THP) chemoprophylaxis of recurrence after transurethral resection of superficial bladder cancer. Hinyokika Kiyo 43: 907-912, 1997 (In Japanese).

5. Turner $\mathrm{N}$ and Grose R: Fibroblast growth factor signalling: From development to cancer. Nat Rev Cancer 10: 116-129, 2010.

6. di Martino E, Tomlinson DC and Knowles MA: A decade of FGF receptor research in bladder cancer: Past, present, and future challenges. Adv Urol 2012: 429213, 2012.

7. Acquaviva J, He S, Zhang C, Jimenez JP, Nagai M, Sang J, Sequeira M, Smith DL, Ogawa LS, Inoue T, et al: FGFR3 translocations in bladder cancer: Differential sensitivity to HSP90 inhibition based on drug metabolism. Mol Cancer Res 12 1042-1054, 2014

8. Bodoor K, Ghabkari A, Jaradat Z, Alkhateeb A, Jaradat S, Al-Ghazo MA, Matalka I, Musleh H and Haddad Y: FGFR3 mutational status and protein expression in patients with bladder cancer in a Jordanian population. Cancer Epidemiol 34: 724-732, 2010.

9. Poyet C, Hermanns T, Zhong Q, Drescher E, Eberli D, Burger M Hofstaedter F, Hartmann A, Stöhr R, Zwarthoff EC, et al: Positive fibroblast growth factor receptor 3 immunoreactivity is associated with low-grade non-invasive urothelial bladder cancer. Oncol Lett 10: 2753-2760, 2015.

10. McConkey DJ, Choi W, Marquis L, Martin F, Williams MB, Shah J, Svatek R, Das A, Adam L, Kamat A, et al: Role of epithelial-to-mesenchymal transition (EMT) in drug sensitivity and metastasis in bladder cancer. Cancer Metastasis Rev 28 : 335-344, 2009.

11. Guancial EA, Werner L, Bellmunt J, Bamias A, Choueiri TK, Ross R, Schutz FA, Park RS, O'Brien RJ, Hirsch MS, et al FGFR3 expression in primary and metastatic urothelial carcinoma of the bladder. Cancer Med 3: 835-844, 2014.

12. L'Hote CG and Knowles MA: Cell responses to FGFR3 signalling: Growth, differentiation and apoptosis. Exp Cell Res 304: 417-431, 2005

13. Katoh $M$ and Nakagama H: FGF receptors: Cancer biology and therapeutics. Med Res Rev 34: 280-300, 2014.

14. Wu GS: Role of mitogen-activated protein kinase phosphatases (MKPs) in cancer. Cancer Metastasis Rev 26: 579-585, 2007.

15. Owens DM and Keyse SM: Differential regulation of MAP kinase signalling by dual-specificity protein phosphatases. Oncogene 26: 3203-3213, 2007.

16. Boutros T, Chevet E and Metrakos P: Mitogen-activated protein (MAP) kinase/MAP kinase phosphatase regulation: Roles in cell growth, death, and cancer. Pharmacol Rev 60: 261-310, 2008.

17. Sun H, Charles CH, Lau LF and Tonks NK: MKP-1 (3CH134), an immediate early gene product, is a dual specificity phosphatase that dephosphorylates MAP kinase in vivo. Cell 75: 487-493, 1993.

18. Li J, Gorospe M, Hutter D, Barnes J, Keyse SM and Liu Y: Transcriptional induction of MKP-1 in response to stress is associated with histone $\mathrm{H} 3$ phosphorylation-acetylation. Mol Cell Biol 21: 8213-8224, 2001.

19. Bang YJ, Jin HK, Kang SH, Kim JW and Yun CY: Increased MAPK activity and MKP-1 overexpression in human gastric adenocarcinoma. Biochem Biophys Res Commun 250: 43-47, 1998.

20. Haagenson KK and Wu GS: Mitogen activated protein kinase phosphatases and cancer. Cancer Biol Ther 9: 337-340, 2010.
21. Loda M, Capodieci P, Mishra R, Yao H, Corless C, Grigioni W, Wang Y, Magi-Galluzzi C and Stork PJ: Expression of mitogen-activated protein kinase phosphatase-1 in the early phases of human epithelial carcinogenesis. Am J Pathol 149: 1553-1564, 1996

22. Manzano RG, Montuenga LM, Dayton M, Dent P, Kinoshita I, Vicent S, Gardner GJ, Nguyen P, Choi YH, Trepel J, et al: CL100 expression is down-regulated in advanced epithelial ovarian cancer and its re-expression decreases its malignant potential. Oncogene 21: 4435-4447, 2002.

23. Wang Z, Xu J, Zhou JY, Liu Y and Wu GS: Mitogen-activated protein kinase phosphatase-1 is required for cisplatin resistance. Cancer Res 66: 8870-8877, 2006.

24. Wang J, Zhou JY and Wu GS: ERK-dependent MKP-1-mediated cisplatin resistance in human ovarian cancer cells. Cancer Res 67: 11933-11941, 2007.

25. Haagenson KK and Wu GS: The role of MAP kinases and MAP kinase phosphatase-1 in resistance to breast cancer treatment. Cancer Metastasis Rev 29: 143-149, 2010.

26. Wang Z, Zhou JY, Kanakapalli D, Buck S, Wu GS and Ravindranath Y: High level of mitogen-activated protein kinase phosphatase-1 expression is associated with cisplatin resistance in osteosarcoma. Pediatr Blood Cancer 51: 754-759, 2008.

27. Zheng L, Chen J, Ma Z, Liu W, Yang F, Yang Z, Wang K, Wang X, $\mathrm{He} \mathrm{D}, \mathrm{Li} \mathrm{L}$ and Zeng J: Capsaicin enhances anti-proliferation efficacy of pirarubicin via activating TRPV1 and inhibiting PCNA nuclear translocation in 5637 cells. Mol Med Rep 13: 881-887, 2016.

28. Kimura T, Suzuki H, Ohashi T, Asano K, Kiyota H and Eto Y: The incidence of thanatophoric dysplasia mutations in FGFR3 gene is higher in low-grade or superficial bladder carcinomas. Cancer 92: 2555-2561, 2001.

29. Ringuette Goulet C, Bernard G, Chabaud S, Couture A, Langlois A, Neveu B, Pouliot F and Bolduc S: Tissue-engineered human 3D model of bladder cancer for invasion study and drug discovery. Biomaterials 145: 233-241, 2017.

30. Camps Mi Nichols A and Arkinstall S: Dual specificity phosphatases: A gene family for control of MAP kinase function.FASEB J 14: 6-16, 2000.

31. Arakawa M, Nakamura K, Yamada Y, Kato K, Katsuda R, Tobiume M,Zennami K, Watanabe M,Kato Y,Nishikawa G, et al: Intravesical administration of pirarubicin against superficial bladder cancer: Relationship between tumor tissue concentration and exposure time in the bladder or therapeutic effect. Exp Ther Med 2: 901-905, 2011

32. Edmondson R, Broglie JJ, Adcock AF and Yang L: Three-dimensional cell culture systems and their applications in drug discovery and cell-based biosensors. Assay Drug Dev Technol 12: 207-218, 2014.

33. Imamura $\mathrm{Y}$, Mukohara T, Shimono Y, Funakoshi Y, Chayahara N, Toyoda M, Kiyota N, Takao S, Kono S, Nakatsura T and Minami H: Comparison of 2D- and 3D-culture models as drug-testing platforms in breast cancer. Oncol Rep 33: 1837-1843, 2015.

34. Li C, Singh B, Graves-Deal R, Ma H, Starchenko A, Fry WH, Lu Y, Wang Y, Bogatcheva G, Khan MP, et al: Three-dimensional culture system identifies a new mode of cetuximab resistance and disease-relevant genes in colorectal cancer. Proc Natl Acad Sci USA 114: E2852-E2861, 2017.

35. Chesi M, Nardini E, Brents LA, Schröck E, Ried T, Kuehl WM and Bergsagel PL: Frequent translocation $\mathrm{t}(4 ; 14)(\mathrm{p} 16.3 ; \mathrm{q} 32.3)$ in multiple myeloma is associated with increased expression and activating mutations of fibroblast growth factor receptor 3 . Nat Genet 16:260-264, 1997.

36. Cappellen D, De Oliveira C, Ricol D, de Medina S, Bourdin J, Sastre-Garau X, Chopin D, Thiery JP and Radvanyi F: Frequent activating mutations of FGFR3 in human bladder and cervix carcinomas. Nat Genet 23: 18-20, 1999.

37. Hernández S, López-Knowles E, Lloreta J, Kogevinas M, Amorós A, Tardón A, Carrato A, Serra C, Malats N and Real FX: Prospective study of FGFR 3 mutations as a prognostic factor in nonmuscle invasive urothelial bladder carcinomas. J Clin Oncol 24: 3664-3671, 2006.

38. Rosenberg JE, Werner L, Bamias A, Choueiri TK, Schutz FAB, O'Brien RSP, Guancial EA, Ross RW, Berman DM, Riester M, et al: FGFR3 protein expression and gene mutation in primary and metastatic urothelial carcinoma (UC) tumors. J Clin Oncol 30 (15 Suppl): S457, 2012

39. Chell V, Balmanno K, Little AS, Wilson M, Andrews S, Blockley L, Hampson M, Gavine PR and Cook SJ: Tumour cell responses to new fibroblast growth factor receptor tyrosine kinase inhibitors and identification of a gatekeeper mutation in FGFR3 as a mechanism of acquired resistance. Oncogene 32: 3059-3070, 2013. 
40. Wu K, Wang B, Chen Y, Zhou J, Huang J, Hui K, Zeng J, Zhu J, Zhang K, Li L, et al: DAB2IP regulates the chemoresistance to pirarubicin and tumor recurrence of non-muscle invasive bladder cancer through STAT3/Twist1/P-glycoprotein signaling. Cell Signal 27: 2515-2523, 2015.

41. Ma G, Pan Y, Zhou C, Sun R, Bai J, Liu P, Ren Y and He J: Mitogen-activated protein kinase phosphatase 1 is involved in tamoxifen resistance in MCF7 cells. Oncol Rep 34: 2423-2430, 2015.

42. Rojo F, González-Navarrete I, Bragado R, Dalmases A, Menéndez S, Cortes-Sempere M, Suárez C, Oliva C, Servitja S, Rodriguez-Fanjul $\mathrm{V}$, et al: Mitogen-activated protein kinase phosphatase-1 in human breast cancer independently predicts prognosis and is repressed by doxorubicin. Clin Cancer Res 15: 3530-3539, 2009.

43. Hu Y, Mintz A, Shah SR, Quinones-Hinojosa A and Hsu W: The FGFR/MEK/ERK/brachyury pathway is critical for chordoma cell growth and survival. Carcinogenesis 35: 1491-1499, 2014.

44. Brondello JM, Brunet A, Pouysségur J and Mckenzie FR: The dual specificity mitogen-activated protein kinase phosphatase-1 and -2 are induced by the p42/p44MAPK cascade. J Biol Chem 272: 1368-1376, 1997.
45. Franklin CC and Kraft AS: Conditional expression of the mitogen-activated protein kinase (MAPK) phosphatase MKP-1 preferentially inhibits p38 MAPK and stress-activated protein kinase in U937 cells. J Biol Chem 272: 16917-16923, 1997.

46. Zhao Q, Shepherd EG, Manson ME, Nelin LD, Sorokin A and Liu Y: The role of mitogen-activated protein kinase phosphatase-1 in the response of alveolar macrophages to lipopolysaccharide: Attenuation of proinflammatory cytokine biosynthesis via feedback control of p38. J Biol Chem 280: 8101-8108, 2005.

47. Takeuchi H, Taoka R, Mmeje CO, Jinesh GG, Safe S and Kamat AM: CDODA-Me decreases specificity protein transcription factors and induces apoptosis in bladder cancer cells through induction of reactive oxygen species. Urol Oncol 34: 337.e11-e18, 2016.

This work is licensed under a Creative Commons Attribution-NonCommercial-NoDerivatives 4.0 International (CC BY-NC-ND 4.0) License. 